\title{
THE MODERN VIEW OF PHYSICAL THERAPY ON RECOVERY OF PATIENTS AFTER STROKE WITH UPPER LIMB SPASTITY
}

\author{
Alona Homola \\ Postgraduate Student, Assistant, National Technical University of Ukraine \\ "Igor Sikorsky Kyiv Polytechnic Institute", Ukraine \\ e-mail: alhzgv@gmail.com,orcid.org/0000-0001-7431-1889
}

\section{Summary}

The purpose of the article is to analyze recommendations for physical therapy methods after acute cerebrovascular accident. Most often, complications of the function of the upper limb occur, this causes difficulties in everyday and professional life. In most cases, the function in the arm is restored worse than in the leg. In case of insufficient restoration of hand function, an occupational therapist teaches the patient the basic skills of self-care: eating, bathing, personal toilet, dressing, controlling bowel movements and urination, using the toilet, moving around, the ability to move on a flat surface, climbing stairs. There are many technical devices that allow you to be independent: an anti-slip mat, prevention of falling in the bathroom, handrails, a step into the bathtub, seats in the bathtub, etc.

In the first month of the recovery period, people after a stroke develop complications contractures of the affected limbs, pain and pulling sensations; spasticity develops, which impairs coordination and balance and, as a result, increases dependence on others. Spasticity increases unevenly, the patient develops a peculiar Wernicke-Mann posture: the upper limb is bent at the elbow and radial wrist joints, the fingers are clenched into a fist; the lower limb is straightened at the hip, knee and ankle joints. Unqualified recommendations and improper selection of a physical therapy program provoke an increase in spasticity.

Objective. To find effective evidence-based physical therapy methods that affect the restoration of upper limb function.

Methods. Scientific literature analysis, synthesis, generalization, pedagogical observation.

An important question for a physical therapist is the selection of effective methods that positively affect the restoration of functional movement of the upper limb, the patient receives an achievable request, and the specialist is able to prevent negative consequences and get good results in a short time.

Keywords: target, recovery, recommendations, hand, contracture, modified Ashworth scale.

DOI: https://doi.org/10.23856/4820

\section{Introduction}

According to the official statistics of the Ministry of Health, in Ukraine, cerebrovascular diseases are the cause of death №2. Every year there are 100,000-110,000 strokes, more than a third of them - in people of working age. 30-40\% of people die within the first 30 days and up to $50 \%$ - within a year from the onset of the disease; $20-40 \%$ of survivors become dependent on outside help, and only about $10 \%$ return to a fulfilling life.

Stroke is an acute disorder of cerebral circulation, characterized by impaired motor, speech and social interactions. 
Factors affecting the risk of stroke: high blood pressure, diabetes mellitus, high blood cholesterol, the presence of bad habits (smoking, excessive alcohol consumption), a sedentary lifestyle, obesity, constant stress. Lifestyle changes can prevent and significantly reduce the risk of stroke.

Thanks to the Uniform clinical protocols of medical care for ischemic, hemorrhagic stroke (scope of examinations, treatment plan and preventive measures), it is possible to prevent negative consequences. The multidisciplinary team builds a common goal and implements recommendations to prevent complications arising in the late period after stroke.

If you choose the right methods of physical therapy in patients after a stroke, the chance of positive dynamics in recovery and prevention of complications such as pressure sores, contractures, pulling pain in the affected limb, and the development of spasticity increases. Patients with spasticity have a loss of sensitivity, impaired balance and coordination, limited range of motion, muscle stiffness, and the inability to be independent in everyday life.

We need a modern view of the problem. Usually the request of patients after stroke: maximum recovery of motor functions. With the help of effective methods of physical therapy, patients after rehabilitation intervention should improve independence in their own body, the ability to use the affected limb and an increase in activity in everyday activities.

\section{Evidence-based methods for upper limb recovery after stroke}

Stroke has consequences at different levels of functioning. Violation of strength, amplitude of movement, leads to a decrease in functioning which affects the quality of life.

Physical therapists must be guided by the ethics of the physical therapist and the job description. Based on human rights in the field of health care, do not harm and carry out rehabilitation measures in the open access for patients who seek help.

Physical therapists work with stroke patients to restore sensorimotor function in the upper and lower extremities. Families and caregivers who are involved in the restoration of functional mobility provide training. Treatment of stroke patients is most effective when all members of the multidisciplinary team are involved. Professionals should understand that the patient is primarily a person who had his own responsibilities, functional capabilities, was responsible every day in the family, work, etc.

Short-term and long-term goal setting should be coordinated with the patient and his family. Each specialist in a multidisciplinary team should make every effort to help restore a person's lost motor functions, give him the opportunity to live independently in order to be socially in demand. Applying the International Classification of Functioning (ICF), which makes it possible to conduct an in-depth analysis of the existing disabilities.

The ICF describes human-related functioning and limitations and serves as a structural basis for organizing information. The ICF combines information into two parts, each of which contains two components (Fig. 1):

Part 1, the component concerns - Functioning and limitation of vital functions: Functions and structure of the organism; Activities and participation.

Part 2 of the component concerns - Contextual factors: Environmental and personality factors (International classification of functions, brief version, 2003).

The international classification of functioning is aimed at restoring lost functions in the opinion of the patient and family, which is necessary for improving functioning, reducing the limitation of vital functions, maximizing the realization of activity and participation. 


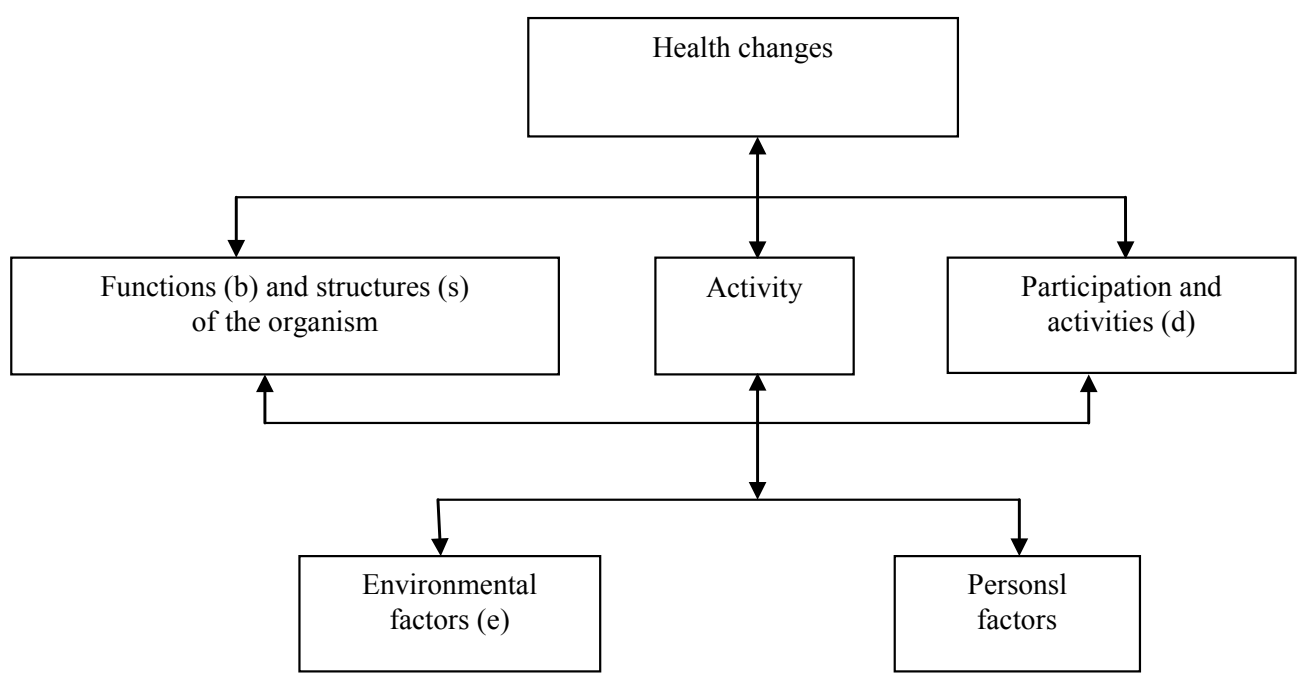

Figure 1. Interaction between the components of the ICF (International Classification of functioning, 2003)

Before starting rehabilitation, it is necessary to study the patient's history, conduct a physical therapy examination, assess the neurological and cognitive state using informative tests and scales.

The Academy of Neurological Physical Therapy, American Physical Therapy Association has developed guidelines for the use of clinical assessment tools in a variety of pathologies, patients in a variety of settings. Chedoke-McMaster Stroke Scale, NIH Stroke Severity Scale, Modified Rankine Scale, Modified Ashworth Scale, Berg Balance Scale (BBS), Visual Analogue Pain Intensity Scale, Arm, Shoulder, and Hand Disability (DASH), the Barthel Index, the Montreal Cognitive Scale (MoCo) and others (Veerbeek et al 2014).

During a general examination, attention is paid to the mode of movement, the nature of movements, the volume of passive movements, the condition of the skin, muscles, ligaments, joints (Potekhin, Zhdanov, 2011).

A neurological examination of the upper limb includes an examination of muscle strength, range of motion and functional capacity of the spastic limb. Tests and scales are used: modified Ashworth spasticity scale, Tardier scale, functional independence scale, test of 9 holes and rods, assessment of the functional activity of the affected limb according to the Frenh scale, and others.

After the examination, the physical therapist learns the patient about the request, goals are planned in SMART format - short-term and long-term (Hertsyk A., 2016). Usually, everyone has a desire, after an acute cerebrovascular accident, to restore movement in the affected limb. An individual rehabilitation program is formed and during each day, the patient and the physical therapist, by the time of discharge, make efforts to achieve the goal.

\section{Analysis of evidence-based medicine methods}

The use of aids: splinting, bandaging, wrist orthoses. The purpose of using orthoses is to reduce spasticity, reduce pain, influence functional results, prevent edema and contractures. 
Treatment indicators were not significant, did not have statistical significance. Bandaging, splinting and the use of orthoses do not improve the motor function of the upper limbs, coordination and accuracy of movements, do not increase the range of motion, do not affect spasticity and muscle strength, and do not reduce edema (Tyso, Kent, 2011). The brace keeps the paretic limb in a passive state, and as a result, it forms contractures and provokes increasing spasticity and pain.

Robotic devices can help patients with active movements, to maintain range and flexibility of movements, and temporarily reduce hypertonicity or resistance to passive movements. The ezoskeleton can help to carry out active movements, but cannot completely perform them independently (Lum et al 2002).

The exoskeleton in combination with therapeutic approaches is ineffective for the rehabilitation of the upper limbs after stroke. Evidence is ambiguous, the ability of the exoskeleton does not improve motor function, accuracy and coordination of movements, does not affect the reduction of spasticity (Mehrholz et al 2012; Mehrholz et al 2015; Veerbeek et al 2014).

The most common forms of virtual stimulators are head-mounted monitors or conventional computer models or projector screens.

The results showed that virtual reality had no significant effect on grip strength or overall motor function. Virtual reality does not improve the function of the upper limb, does not affect the coordination of movements, and has no effect on reducing spasticity, accuracy and muscle strength (Laver et al., 2015).

Functional electrical stimulation is used for the upper limb in hemiparetic lesions. In two studies, electrical stimulation was performed in combination with exercise therapy, and the program was based on exercise therapy. There were no significant differences between the groups (Hsu et al., 2010, Kowalczewski et al., 2007).

Using neuromuscular electrical stimulation that does not increase muscle strength and does not improve motor function. The literature provides mixed data on reduced spasticity and increased range of motion or improved outcomes after stroke severity (Kwakkel et al 2016).

Analysis of materials does not provide effective methods for restoring the function of a spastic upper limb. It is advisable to continue to study and research a topic that remains open to the consideration of scientists: physical therapy of patients after stroke with spasticity of the upper limb in the late period.

With the help of physical therapy methods, patients after a stroke with spasticity of the upper limb in the late period have the opportunity to restore the function of the affected limb for further use in domestic activities.

\section{Pain in the shoulder after a stroke}

One of the consequences of a stroke that leads to disability is shoulder pain (Najenson et al., 1971). The hand in the initial period with hemiplegic form is hypotonic or sluggish. The cause of the subluxation of the shoulder is not the stability of the rotator cuff of the muscle-tendon sleeve. The muscles of the shoulder do not perform their function, they do not contain the head of the humerus in the glenoid fossa. Also proper care and maintenance in bed, lack of support in an upright position contribute to shoulder subluxation (Chaco, Wolf, 1971)

The main factor in shoulder pain is spasticity, which is most common in patients with hemiplegia. Tense muscles inhibit movement, decrease amplitude, and muscle imbalance occurs. Contraction of all muscles located around the shoulder joint: shoulder adductors, subscapularis, pectoralis major, round and latissimus dorsi. 
Stable support and static shoulder stretching are considered insufficient to reduce pain, and neuromuscular electrical stimulation has not been proven to be effective.

\section{Conclusion}

In the process of physical therapy, it is advisable to use methods that are aimed at improving the range of motion, reducing pain and improving the patient's muscular activity. Methods that are aimed at developing gross and fine motor skills, balance and coordination of movements. With the help of effective methods of physical therapy, as a result, an appropriate stereotype of movements is formed, does not have compensatory defects and, as a result, does not return the affected limb to the previous intolerable state.

After analyzing the methods of evidence-based medicine, we came to the conclusion that it is advisable to study the topic: physical therapy of patients after a stroke with the introduction of effective methods to help restore the lost motor function in the upper limb.

\section{References}

Chaco J., Wolf E. (1971). Subluxation of the glenohumeral joint in hemiplegia. American journal of physical medicine \& rehabilitation. 50 (3), 43-139

Hertsyk A. (2016). Smart goal setting in physical therapy. Fizvykhovannia, sport i kultura zdorovia u suchasnomy suspilstvi. 2 (34), 16-57

Hsu S.S., Hu M.H., Wang Y.H., Yip P.K., Chiu J.W., Hsieh C.L. (2010). Dose-response relation between neuromuscular electrical stimulation and upper-extremity function in patients with stroke. Stroke. 41 (4), 821-4

International classification of functions, brief version. (2003). International Classification of functioning, limitations of daily living and health. World Health Organization. P 228. [Saint Petersburg]

Kowalczewski J., Gritsenko V., Ashworth N., Ellaway P., Prochazka A. (2007). Upper-extremity functional electric stimulation-assisted exercises on a workstation in the subacute phase of stroke recovery. Archives of physical medicine and rehabilitation. 88 (7), 9-833

Kwakkel G., Winters K., Van Wegen E., Nyland R. H., Van KuikA. A., Visser-Meili A., De Groot J., De Vlugt E., Arendzen J. H., Geurts AS, Meskers K. (2016). Effects of unilateral upper limb training in two different prognostic groups at the onset after stroke: a randomized clinical trial of explicit-stroke. Neurorehabilitation and neurorepair. 30 (9), 16-804

Laver K., George S., Thomas S., Deutsch J., Crotty M. (2015). Virtual Reality for Stroke Rehabilitation: An Abridged Cochrane Review. Euro. J. Phys. Rehabil. Med. 51 (4), 497-506

Lum P., Reinkensmeyer D., Mahoney R., Reimer V.Z., Burgar S. (2002). Robotic devices for movement therapy after stroke: current status and challenges for clinical acceptance. Stroke rehabilitation topics. 8 (4), 40-53

Mehrholz J., Hädrich A., Platz T., Kugler J., Pohl M. (2012). Electromechanical and robotic arm training to improve daily activity, arm function and arm muscle strength after stroke. Cochrane Database of Systematic Reviews, 172-173

Mehrholz J., Pohl M., Platz T., Kugler J., Elsner B. (2018). Electromechanical and robotic arm training to improve daily life, arm function and arm muscle strength after stroke. Cochrane Database Syst. Rev, 9 (9)

Najenson T., Yacubovich E., Pikielni S.S. (1971). Rotator cuff injury in shoulder joints of hemiplegic patients. Scandinavian journal of rehabilitation medicine. 3 (3), 7-131 
Potekhin L.D, Zhdanov V.N (2011). Rehabilitation of disabled people with impaired motor functions. Abstracts. "Quality of life" - a complex indicator of the social status of disabled people. Part 1, P. 38-40. [Novokuznetsk].

Robert Teasell, M.D, Norhayati Hussein, M.D, Magdalena Mirkowski, MSc, MScOT, Danielle Vanderlaan, RRT, Marcus Saikaley, HBSc, Mitchell Longval, BSc, Jerome Iruthayarajah, (2021). Rehabilitation of the upper clinical course in the case of hemiplegic form of the disease. International Neurological Journal, 1 (17)

Tyson S.F., Kent R.M. (2011). The effect of upper limb orthopedics after stroke: a systematic review. Neurorehabilitation. 28 (1), 29-36

Veerbeek J.M., Wegen E., Peppen R., Wees P.J., Hendriks E., Rietberg M., Kwakkel G. (2014). What is the evidence for physical therapy poststroke? A systematic review and meta-analysis. PloS one. 9 (2), doi.org/10.1371/journal.pone.0087987 